\title{
CONJUGATE HEAT TRANSFER STUDY OF A CENTRIFUGAL COMPRESSOR WITH IMPELLER CAVITIES
}

\author{
Maurice Stahl \\ MAN Energy Solutions SE \\ Maurice.Stahl@man-es.com \\ Oberhausen, Germany
}

\author{
Dr.-Ing. Holger Franz \\ MAN Energy Solutions SE \\ Holger.Franz@man-es.com \\ Oberhausen, Germany
}

\author{
Dr.-Ing. Lutz Itter \\ MAN Energy Solutions SE \\ Lutz.Itter@man-es.com \\ Oberhausen, Germany
}

\begin{abstract}
In this study the effects of heat transfer on the rotorstator cavity temperatures and the characteristics of one industrial, single stage centrifugal compressor are examined. A baseline Computational Fluid Dynamics (CFD) analysis of the fluid with adiabatic (AD) boundary conditions is conducted. The relevant solid bodies are added to the model and a Conjugate Heat Transfer (CHT) calculation of the machine is performed. The two models are compared and validated by experimental data.

Most research regarding heat transfer in centrifugal compressors focusses on turbocharger or gas turbine applications, where a significant heat flux from the adjacent turbine is expected. Industrial compressors are often treated as adiabatic. Some publications include the solid body of the impeller in the calculation, but cavities are often not in the focus of the study. Efforts to obtain reliable Heat Transfer Coefficients (HTC) by employing simple correlations for the impeller flow in the presence of a shroud cavity leakage have not yet proven to be successful.

When comparing the CHT and the adiabatic model, differences can be observed in wall- and cavity flow temperatures. The adiabatic approach exceeds the measured cavity temperatures by $40 \%$, whereas the CHT calculation yields significantly better results and stays within a margin of error of $1-4 \%$. Additionally, the qualitative temperature distribution and calculated frictional power loss of both models differ noticeably.

The bulk flow seems nearly unchanged and the adiabatic approach proves to be sufficient to describe overall compressor performance. Differences in efficiency, total pressure ratio and total temperature ratio between the two models are negligible.

For machines engineered to maximum mechanical loads, with tight restrictions in fluid temperature, or if thermal expansion of the components is critical (e.g. tight sealing clearances), a CHT simulation is recommended for more accurate temperature predictions.
\end{abstract}

\section{INTRODUCTION}

The optimal design strategy for turbomachinery components requires a good understanding of the thermoand fluid-dynamical processes. Of equal interest are the structural-mechanical properties. Both fields are influenced by the fluid- and solid body temperatures. In some industrial applications of centrifugal compressors, certain gas temperatures must not be exceeded to prevent exothermal decay of the gas. From a mechanical viewpoint, the solid temperatures greatly influence material properties, strain, stress and therefore the structural integrity of the design.

Given the right boundary conditions, modern CFD codes are, within a certain degree of accuracy, capable of predicting the temperature distribution in the flow. But obtaining the right boundary conditions is critical.

Adiabatic walls represent a widely used assumption in comparatively low temperature applications like centrifugal compressors. CHT calculations, which are often used in high temperature environments such as gas turbines or turbochargers, can shift the problem: By including the solid bodies in the CFD calculation, heat transfer through the bodies eliminates the need for temperature or heat flux assumptions at fluid wall boundaries. But modelling effort increases and valid boundary conditions at external solid walls are still necessary.

(Bohn et al., 2005) show that a full CHT calculation of a centrifugal compressor in a turbocharger has significant influence on the compression process and efficiency.

(Borm et al., 2011) include the solid body of the impeller in a CHT calculation. Only a small impact on efficiency was found. In both studies, cavity flows were not extensively researched.

By comparison with a full CHT including cavity modelling (Kluck et al., 2006) found that simple 1D correlations for HTCs are often not valid and that secondary flows complicate heat transfer predictions. 
(Lei et al., 2007) introduced a so called "fast CHT" method, by connecting a 1D flow solver to a solid body model. The body temperatures of a previous full CHT calculation could be approximated, but cavity leakages were not further researched.

Literature shows that the inclusion of rotor-stator cavities is crucial to the correct reproduction of stage characteristics:

(Hu et al., 2017) and (Sun et al., 2009), amongst others, research centripetal flow through rotor-stator cavities and show how axial thrust and disk torque are affected.

(Guidotti et al., 2012) show the influence of cavity modelling on the efficiency and pressure ratio.

(Mischo et al., 2009) find that the shroud cavity leakage can induce flow separation and thereby severely influence compressor performance.

In most studies, the walls are modelled as adiabatic. But especially in rotor-stator cavities, where disc friction heats up the flow, this simplification yields comparatively high temperatures since heat can only be transported over domain boundaries by a through-flow.

The aim of this study is to examine the influence of a CHT calculation on the cavity temperatures in an industrial centrifugal compressor and the effects on wall temperatures and compressor performance.

\section{METHODOLOGY}

\section{Experimental Setup}

The measurement of the examined centrifugal compressor stage was conducted back in 2011. The experimental data was collected during the development phase of a new stage design. It was chosen for this paper due to the fact that cavity temperatures were measured and an impact of cavity flow on the stage performance was suspected.

The testing facility itself is located in a closed factory building and contains separate housings for machine evaluation. Figure 1 shows a cross section of the compressor with probe positions and probe types.

Air radially enters the machine through the first row of stationary inlet guide vanes (IGV) and passes a second row of potentially adjustable IGVs. The shrouded impeller (LA) is equipped with stepped labyrinth seals on top of the shroud disk. A bladeless, vertical plate diffuser was used in this test run. The cavity behind the impeller hub (rear cavity) is connected to the bladed return channel (RC) via a seethrough labyrinth seal. While the enclosure is (ideally) disconnected from the gas path, the bottom cavity is connected to the return channel and to the rear cavity of the impeller. The gas exits the machine axially.

The inlet cavity at the impeller hub is adjacent to a ballbearing. The bearing is only installed for safety reasons and is unloaded during the testing. Therefore, no significant heat flux is expected.

Total temperature, static temperature, total pressure and static pressure were measured at various positions and by multiple redundant probes in the flow. Additionally, rotational speed was measured, and other data was collected to compute volume flow and input torque.

The stage is designed for the circumferential Mach number $M a_{u}=0.87$ and the flow number $\varphi_{I, t o t}=0.12$. The following definitions are used:

$$
\begin{aligned}
M a_{u} & =\frac{u_{2}}{a_{s, I, t o t}} \\
\varphi_{I, t o t} & =4 \cdot \frac{\dot{V}_{I, t o t}}{\pi \cdot D_{2}^{2} \cdot u_{2}}
\end{aligned}
$$

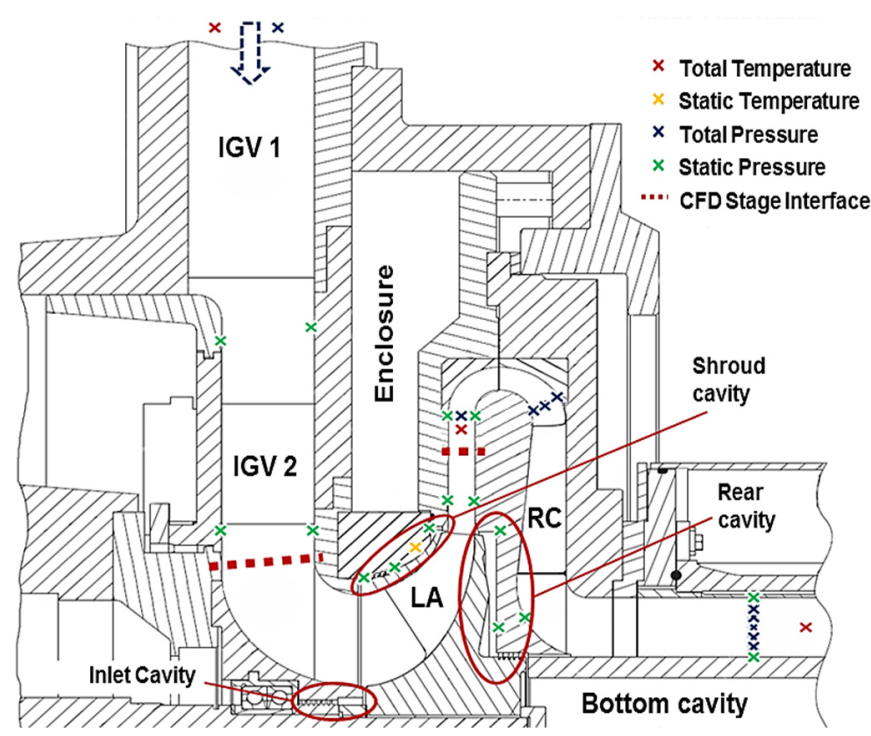

Figure 1 Cross Section of Compressor with Probe Positions and Type

\section{CFD / CHT Model}

The complete fluid mesh was created in Numeca Autogrid 12.2, but Ansys CFX 18.2 was used for the solid mesh creation, pre- and postprocessing, as well as for the solution.

\section{Geometry}

The complete, as built, 3D geometry is available from the design process, but is further simplified: All holes are closed and small features, as well as fillet radii on impeller and return channel blades are removed. Additionally, probes and other non-axisymmetric bodies are excluded from the model. The ball bearing is modelled as a solid body, to allow heat transfer from the rotor to the inlet contour.

The fluid domains are split into the IGVs $(1 \& 2)$, the impeller and the return channel. Three rotor-stator cavities at the inlet, the shroud and the back of the impeller are also modelled. While the bottom cavity is included in the model, the enclosure is not. Of each blade row, only one axisymmetric segment with correct pitch is modelled. The position of the stage interfaces (mixing-plane) is indicated by dotted red lines in Figure 1.

The solid bodies are created as a full-matching, axisymmetric geometry. Some solid parts were neglected to reduce the model size. Figure 2 shows the complete CHT setup and external boundary conditions. 


\section{Boundary Conditions}

Solid bodies in Figure 2 are coloured according to their domain affiliation: Since the impeller and the corresponding rotor-stator cavities are calculated in the rotating frame of reference, the adjacent solids are split up horizontally and vertically. This allows a better definition of the interfaces. At every contact surface an interface handles the heat transfer between the different bodies. Since rotational periodicity is defined for all domains, the automatic pitch change option is used to connect domains with different pitch values.

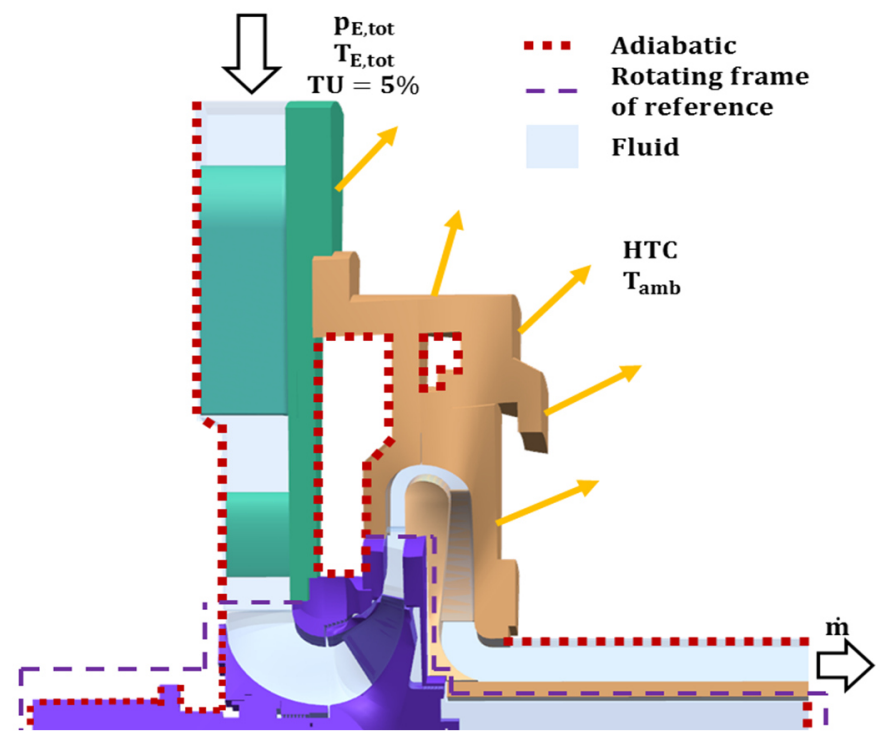

\section{Figure 2 Complete CHT Setup with Fluid and Solid Bodies}

The external surfaces of the machine are subject to natural convection inside the housing. Therefore, the external surfaces of the casing are constrained by an HTC and the ambient temperature of the housing, which was measured. The different HTCs for the cylindrical (Churchill et al., 1975) and vertical circular (Lewandowski et al., 2001) surfaces are obtained with Nusselt numbers $(\mathrm{Nu})$ of the forms:

$$
\begin{aligned}
& N u_{c y l}=\left\{0.725+0.387 \cdot[\mathrm{Gr} \cdot \mathrm{Pr} \cdot 0.345]^{1 / 6}\right\}^{2} \\
& N u_{c i r c}=0.655 \cdot(\mathrm{Gr} \cdot \mathrm{Pr})^{1 / 4} \\
& G r=\frac{l_{c}^{3} g}{v^{2}} \beta_{v} \Delta T
\end{aligned}
$$

$G r$ is the Grashof number and $P r \approx 0.70$ the Prandtl number, which is already included in the equations and, for simplicity reasons, assumed to be constant for the external HTC calculation. The characteristic length for the cylindrical- and the vertical surfaces are defined differently:

$$
\begin{aligned}
& l_{c, c y l}=\frac{1}{2} \cdot \pi \cdot d_{c y l} \\
& l_{c, c i r c}=d_{c i r c}
\end{aligned}
$$

The circular enclosure is defined as adiabatic. A simplified test with correlations for rectangular enclosures (Berkovsky and Polevikov, 1977) was conducted and did not show a significant impact on the solution. It is therefore neglected.

To simplify the comparison of both models, thermal strain was not regarded for seal gap calculation. Thus, labyrinth seals have only been adjusted to match the seal gap at rotational speed. The remaining geometry was modelled at cold state.

\section{Mesh}

Special focus was put onto the rotor-stator-cavity discretization. Exemplary, Figure 3 shows the meridional mesh of the shroud cavity's labyrinth seal.

A low-Reynolds mesh with 16 million hexahedral cells, boundary layer expansion ratios $\leq 1.2$ and $y^{+} \leq 1$ was chosen for the final fluid setup. The differences in cavity temperatures compared to the finest created mesh with about 40 million cells are less than $0.3 \%$. The differences to other values of interest to the finest mesh are shown in the appendix (Fig. C.1).

The solid bodies were either meshed with unstructured tetra elements or, when possible, with hexahedral elements. It was ensured that all thin bodies are covered by at least four to five elements. Additionally, the surface mesh was refined on contact surfaces to reduce numerical error at the nonmatching general grid interfaces.

The adiabatic model contains the same fluid mesh, without the solid bodies. Every surface (with some exception) is constrained to be adiabatic. The large cavity under the return channel and the small cavity under the inlet contour are constrained by a constant temperature boundary condition: Since both cavities have no noteworthy throughflow, temperature levels would otherwise rise indefinitely in an adiabatic calculation. The inlet cavity is constrained by the total temperature at the inlet. The cavity under the stage outlet is constrained with the total temperature at the outlet. This assumption agrees well with the CHT results.

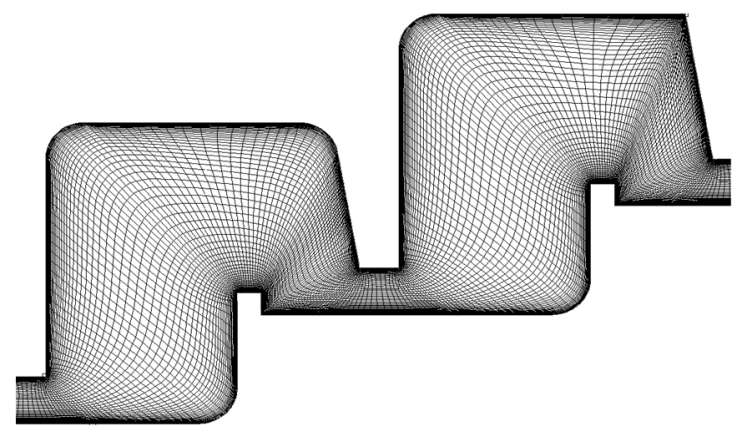

Figure 3 Meridional Mesh of the Labyrinth Seal of the Shroud Rotor-Stator Cavity

\section{Computational Setup}

For every probe position in Figure 1 and in every solid body, a Monitor Point was configured to observe the solution process and to judge convergence levels. All imbalances were also monitored until at or below $0.01 \%$.

The complete setup was solved in CFX 18.2 steady state with the SST-k $\omega$ turbulence model. Additionally, the Curvature Correction and the Reattachment Modification 
were used to improve off-design behaviour. The SST-model is known to handle separation in adverse pressure gradients, while having the advantages of a turbulent viscosity model regarding computational effort and stability. In other publications, the model has shown good agreement with measurements in rotor-stator cavities (Hu et al., 2017; Barabas et al., 2015) - the primary focus of this study.

An initial solution for the calculation was established with ideal gas properties of air and frozen rotor interfaces. Stage interfaces (mixing plane) as well as temperature and pressure dependent air properties according to VDI Heat Atlas data (Span, 2006) were configured and used for all further calculations. The total energy equation was used with a manually adjusted time step to allow significantly faster convergence of the temperature levels in both models.

\section{RESULTS AND DISCUSSION}

\section{General Compressor performance}

For evaluation of the general model validity, the results of the calculations are compared to experimental data of the compressor main flow.

Especially the values for low flow numbers show good agreement with the measurements; calculated values are consistently slightly higher than in the experimental data and reproduce the characteristics of the machine. Since fillet radii and other flow obstructions were removed, deviations towards higher flow numbers are acceptable.

Figure 4 and Figure 5 show the normalized total outlet temperature and total outlet pressure over flow number. Normalization was done with the respective overall maximum value.

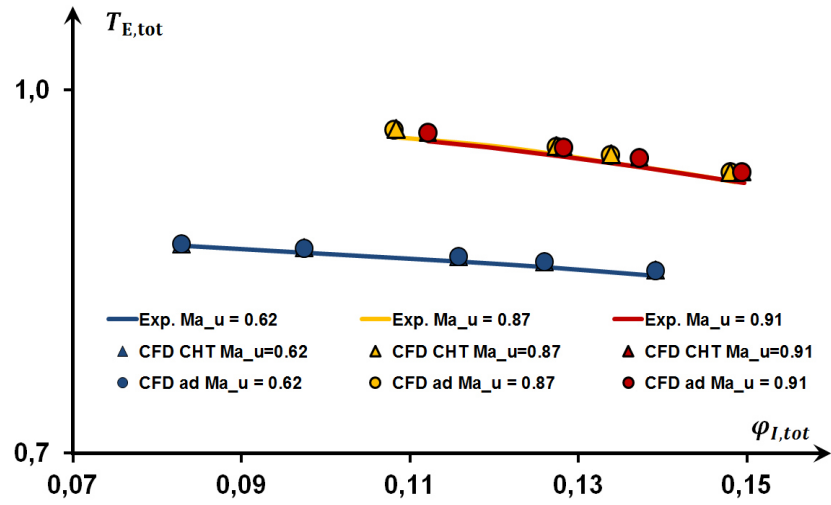

Figure 4 Normalized Total Outlet Temperature at Probe over Flow Number (AD/CHT/Experimental)

Between both models no differences in total pressure ratio, total temperature ratio and efficiency larger than $0.2 \%$ can be observed. The resulting external heat flux over the outer casing is a fraction $(0.1-0.4 \%)$ of the shaft work input.

The primary effect of the CHT calculation is the redistribution of thermal energy inside the system, especially regarding rotor-stator cavities. The inlet cavity at the impeller hub (Figure 1) is not further discussed, since no significant influence on the main flow can be seen. Therefore, the focus is put on the shroud and rear cavity.

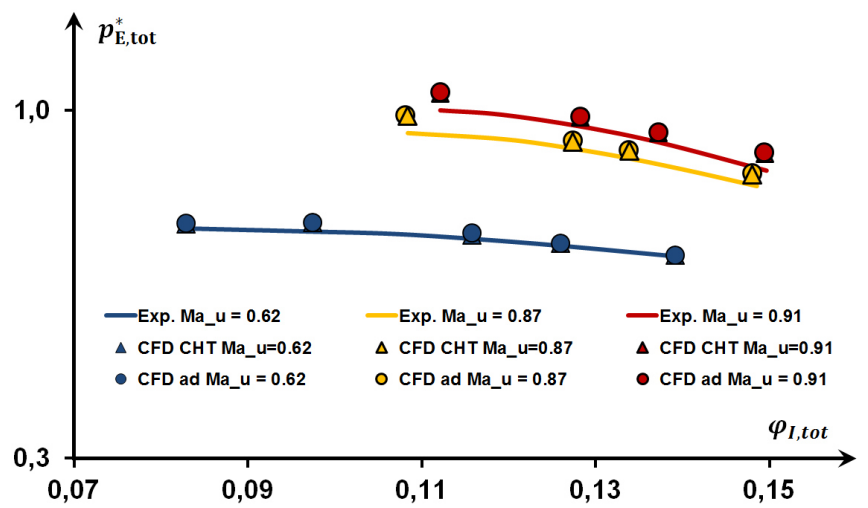

Figure 5 Normalized Total Outlet Pressure at Probe over Flow Number (AD/CHT/Experimental)

\section{Rotor-Stator Cavities}

\section{Flow Structures and Heat Transfer Mechanisms}

The rotor-stator cavities in this study are, by turbulence model definition, fully turbulent and within a relatively large geometrical gap. Rotor and stator boundary layers are mostly separated by a rotating core flow. This represents a "Regime IV flow" according to (Daily et al., 1960).

Figure 6 shows that in both cavities, the circumferential velocity dominates over superposed meridional flows that develop after the cavity inlets, when the flow becomes rotationally periodic $(\mathbf{A}, \mathbf{1})$. At the rotor walls, the fluid is forced upwards by centrifugal forces $(\mathbf{B}, \mathbf{2})$. At the casing walls, the radial flow direction is negative, pointing towards the axis of rotation $(\mathbf{C}, \mathbf{3})$. Additionally, a recirculation occurs that transports heat from rotor to stator side $(\mathbf{D}, \mathbf{4})$. The flow structures are identical in the adiabatic and in the CHT model.

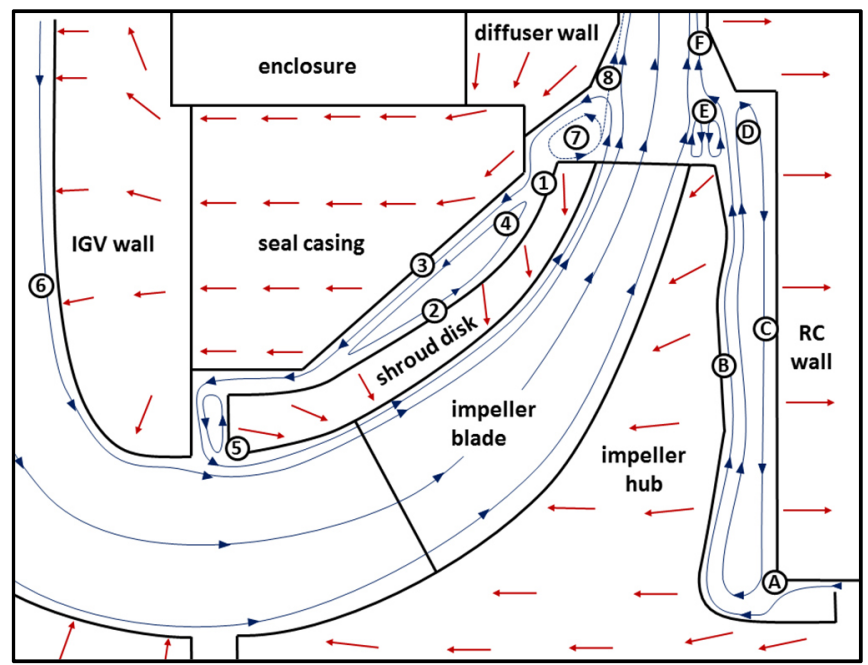

Figure 6 Qualitative Meridional View of Main Flow Structures and Heat Transfer Mechanisms

In the rear cavity, leakage with very low pre-swirl enters at a low radial position (A) - the hub labyrinth seal - and is 
accelerated upwards at the impeller wall (B). The re-entering of the leakage into the compressor main flow is characterized by strong swirling over the edge of the impeller $(\mathbf{E})$, before re-entering mainly on the suction side of the blade $(3 \mathrm{D}$ effect) (F).

The flow at the shroud cavity's inlet (1) is characterized by a pre-swirl originating from the impeller main flow. An eddy over the blunt edge of the shroud disk (7) blocks most of the cavity's inlet. As a result, the leakage flow on the pressure side of the blade is forced into the cavity at the casing wall while a portion recirculates around the eddy (7). On the blades suction side, a partial amount of the previously circulating flow re-enters the main flow path (8). Therefore, two heated streaks of leakage pass along the diffuser and the return channel walls $(\mathbf{F}, \mathbf{8})$.

The remaining leakage of the shroud cavity descends at the seal casing (3) and exits at the impeller inlet (5). It continues to flow alongside the shroud disk. As a result, the convective heat transport in the cavities strongly depends on leakage direction and mass flow.

\section{Heat Flux}

For the CHT model, the direction of the heat flux density inside the surrounding solid bodies (represented by red arrows in Figure 6) can be derived from the body temperatures and Fourier's law:

$$
\dot{\vec{q}}=-\lambda_{S} \cdot \nabla T
$$

Thermal energy in the shroud cavity is transported to the main flow through the shroud disk, the impeller blade and the seal casing. This results in a pre-heating effect of the flow at the IGV wall (6). The rear cavity is primarily cooled by the impeller hub and secondarily by the return channel wall.

Due to rising temperatures towards the impeller outlet, the temperature difference between the main flow and the cavities decreases. At the shroud cavity outlet (5) the hot, reentering leakage impedes heat flux. Therefore, the heat flux is directed towards central streamwise locations of the impeller (exaggerated in Figure 6, for illustrative purposes).

The heat flux parallel to the enclosure wall is evidence of the adiabatic wall boundary condition.

The ratio of heat flux from the cavities to the casing walls vs. the heat flux from the cavities to the impeller walls ( $\dot{\mathbf{Q}}_{\text {Casing }} / \dot{\mathbf{Q}}_{\text {Rotor }}$ ) increases with flow number (Figure 7). Thus, the importance of heat flux through casing walls is not negligible and increases further with flow number.

This effect is more dominant in the shroud cavity, where the heat flux to the casing gets significantly higher compared to the shroud disk. The heat flux to the casing (from the shroud cavity to the IGV) increases due to higher Reynolds numbers in the IGV and therefore higher HTCs. Additionally, the casing is made of Aluminium with an almost 10 times higher thermal conductivity than the steel rotor. At the same time the heat flux to the rotor decreases, as (averaged) HTCs under the impeller shroud disk decrease slightly with flow number on the same speed-line. This correlates with a decrease in eddy viscosity in this region towards higher flow numbers.
In the rear cavity, the heat flux to the impeller hub dominates. This is due to a larger Temperature difference between the rear cavity and the colder impeller main flow; the temperature difference between the rear cavity and the comparatively hotter return channel is smaller. Thus, the heat flux to the impeller hub is more than twice as big as the heat flux to the casing, but still the ratio increases with flow number. For the rear cavity, this can be attributed to the decreasing temperature difference between the cavity and the return channel towards lower flow numbers. The Impeller flow, despite having higher discharge temperatures at lower flow numbers, still enables conduction to the cold inlet flow.

The ratios of heat flux prove that the impeller- and adjacent casing bodies (especially those that enable thermal conduction to the colder stage inlet) are crucial for accurate cavity temperature predictions.

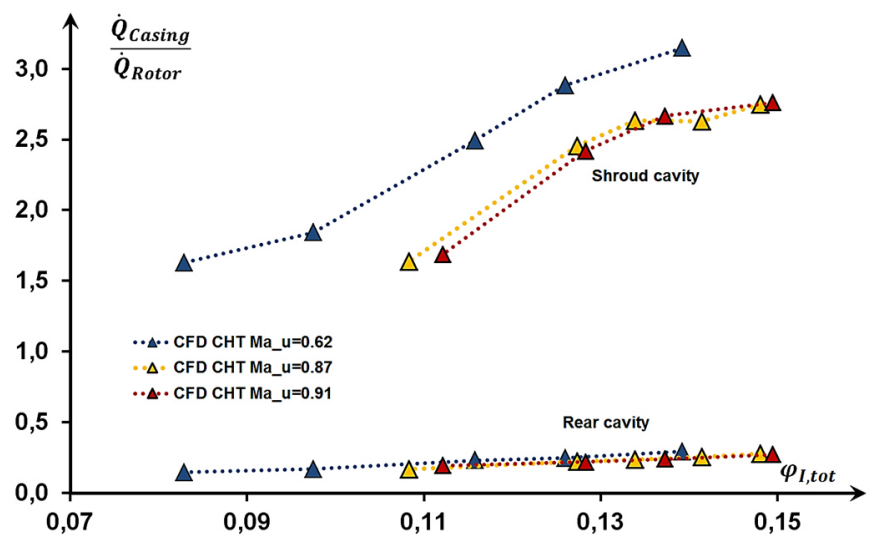

Figure 7 Ratio of Heat Flux to Casing vs. Heat Flux to Impeller (Shroud cavity)

\section{Temperature Distribution}

\section{Rotor-Stator Cavities}

Temperature differences between the two models can be observed in the cavity temperatures. Figure 8 and Figure 9 show the respective static flow temperatures. They are normalized by the same maximum (Celsius) temperature of the adiabatic calculation but shown on different (!) colour scales ( 1 vs. 0.7$)$ to enable a better visual representation of qualitative temperature distribution.

In the rear cavity, the leakage flow is heated by disk friction at the rotor side and therefore transports thermal energy radially upwards. The flow recirculation inside the cavity (Figure 6, D) transports a part of that energy axially towards the casing side and radially downwards. This results in an accumulation of thermal energy towards the casing wall at higher radial positions (Figure 8 and 9, C).

The rear cavity temperature distribution of the CHT model is qualitatively similar to the one of the adiabatic model but maximum temperatures are $30 \%$ lower (mind the different scales of Figures 8/9).

In the shroud cavity, the ascending portion of recirculating flow at the rotor wall is heated at the shroud disk. At the impeller outlet this leads to locally higher wall temperatures at the disk surface (Figure 8/9, B). The 
descending leakage transports the additional thermal energy downwards to the labyrinth seal. Thus, in the adiabatic model, thermal energy accumulates at lower radial positions (Figure 8, A).

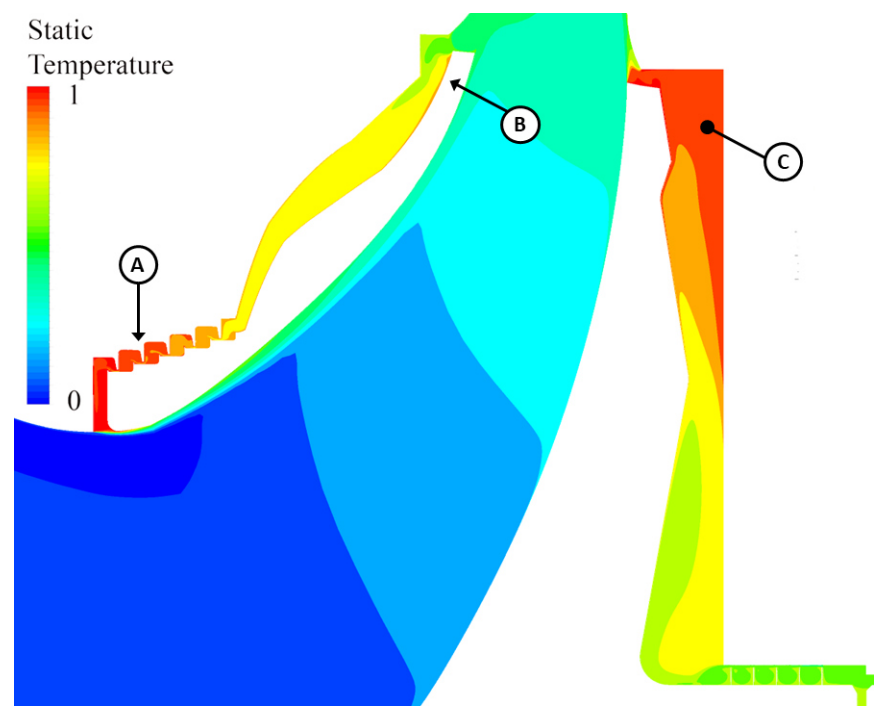

Figure 8 Dimensionless Static Flow Temperatures in Impeller and Rotor-Stator Cavities at $M a_{u}=$ $0.87, \varphi=0.14$ (AD -scale: $\max 1.0$ )

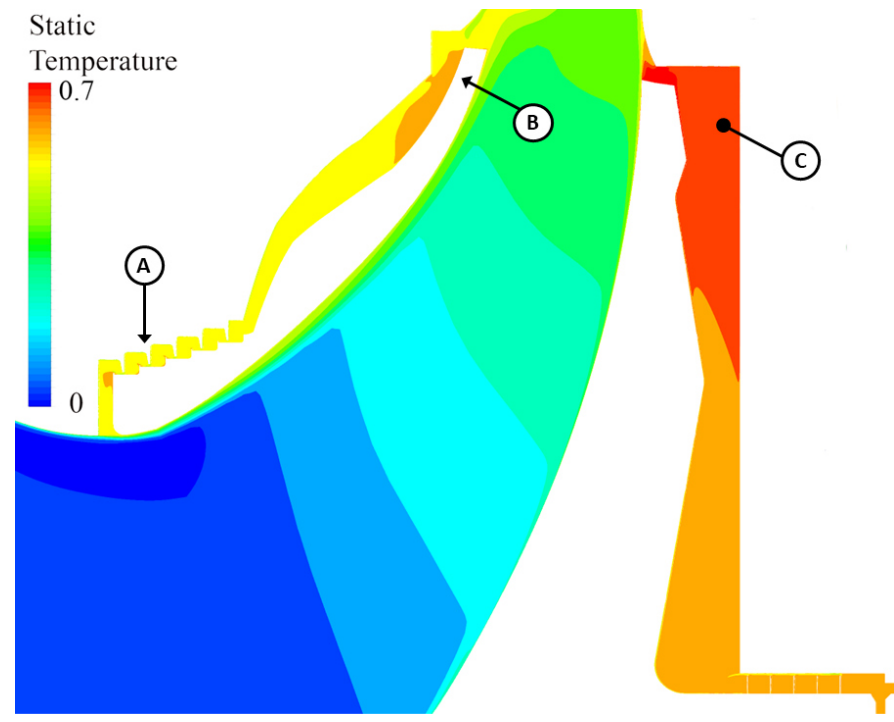

Figure 9 Dimensionless Static Flow Temperatures in Impeller and Rotor-Stator Cavities at $M a_{u}=$ $0.87, \varphi=0.14$ (CHT - scale: $\max 0.7)$

The CHT model cools the leakage flow via the shroud disk and seal casing towards lower radial positions (Figure 9, A).

Especially in the upper part of the rear cavity and in the lower part of the shroud cavity the adiabatic model yields about 1.5 times higher static temperatures than the full CHT model (compare Figures 8/9, $\mathrm{A}$ and $\mathrm{C}$ ). In these regions, no experimental data is available to evaluate the accuracy of the models. At the static temperature probe location, large differences can be observed. The comparison of static shroud cavity flow temperatures, of both models, with experimental data is shown in Figure 11.

Compared to the measurements, the adiabatic model consistently predicts the cavity flow temperatures about $40 \%$ (Celsius scale) too high. The CHT model shows noticeably reduced temperatures and better agreement to the experimental data. Static temperatures are calculated 1-4\% (on Celsius scale) higher compared to experimental data.

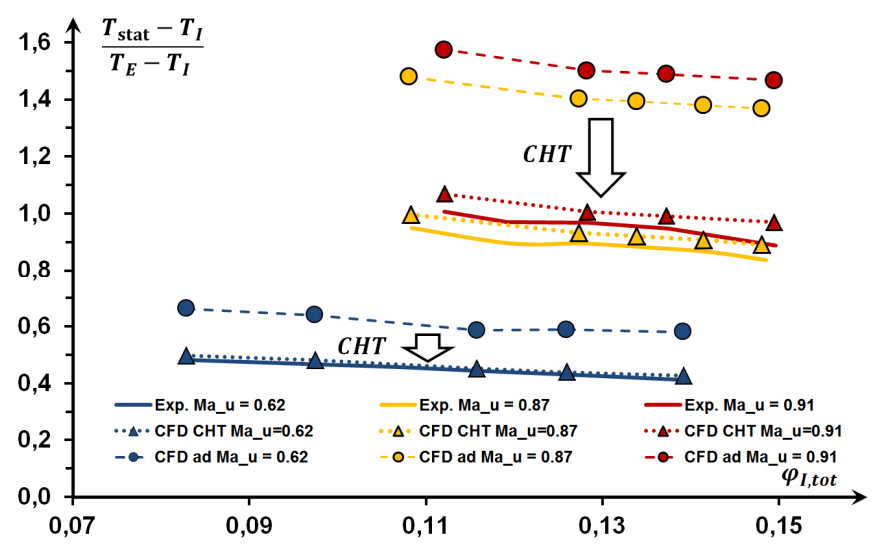

Figure 11 Normalized Static Flow Temperature at Shroud Cavity Probe over Flow Number (AD / CHT / Experimental)

\section{Main Flow}

Figure 12 presents the effects on impeller main flow temperatures. The bulk temperature is only slightly affected by additional heat transfer. While the adiabatic model consistently over estimates wall temperatures in the cavities, it under estimates those in the main flow; wall temperatures in the main-flow are considerably higher in the CHT model. This is especially evident upstream of the shroud leakage and before the impeller outlet.

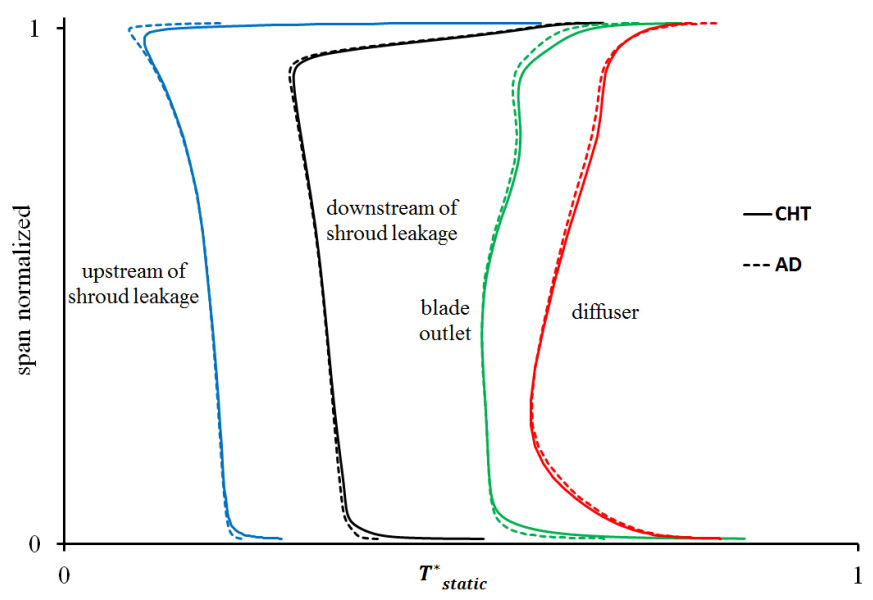

Figure 12 Circumferentially Averaged Normalized Static Flow Temperature in the Main Channel at $M a_{u}=0.87, \varphi=0.14(\mathrm{AD} / \mathrm{CHT})$

Upstream of the shroud leakage, in the IGV section, the thermal boundary layer is influenced by the heat flux from the shroud cavity and the rotor. The wall temperature of the 
adiabatic model is lower, but almost immediately rises after the shroud leakage re-enters the main flow. At the shroud cavity outlet, hot leakage mixes with the main channel flow, which was already pre-heated in the IGV section. After an initial mixing process, both models predict similar temperature contours under the shroud. In the diffuser, differences in wall temperatures are much smaller.

Velocity and pressure in the main flow are nearly unchanged and are therefore not presented.

At the impeller outlet, high cavity temperatures (Figure $\mathbf{8} / \mathbf{9}, \mathrm{C})$ raise the wall temperatures through conduction (CHT). This influences the blade wall temperatures, which can be seen in Figure 13.

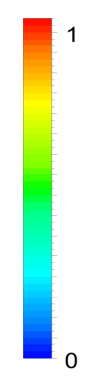

\section{Wall Temperature (identical scale)}
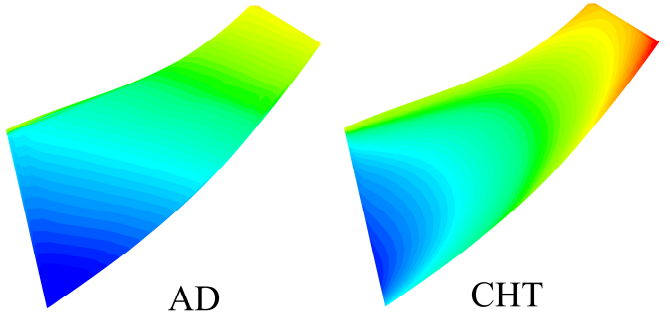

Figure 13 Comparison of Wall Temperatures on the Impeller Blade at $M a_{u}=0.87, \varphi=0.14(\mathrm{AD} / \mathrm{CHT})$

The adiabatic model yields a more uniform, nearly equiradial, temperature distribution, while the CHT model reveals the influences of cavity temperatures: Hub and span of the blade show higher Temperatures (CHT), while the cooling effect of the main flow dominates at 0.5 span. Therefore, at center span, streamwise temperature distributions are similar.

In Figure 14 the wall temperatures of the impeller are presented on identical scale (upper part) and on local scales (lower part).
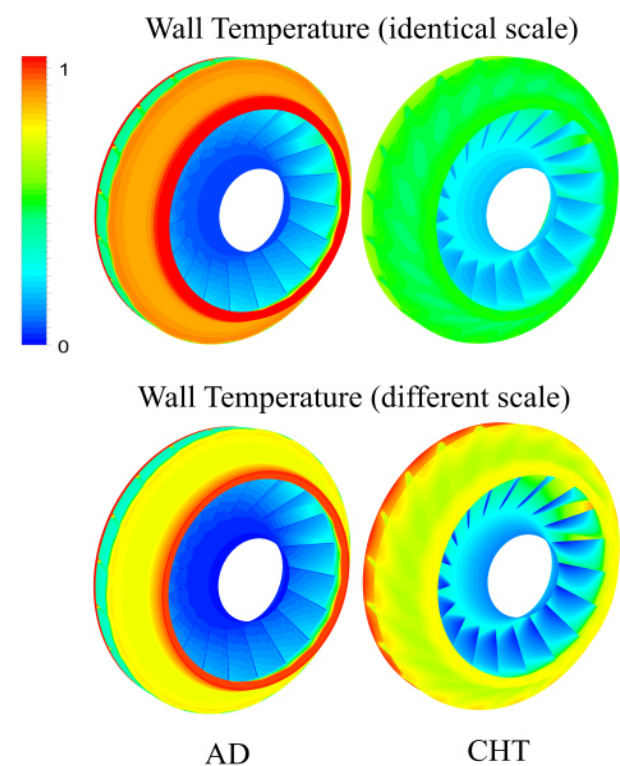

Figure 14 Comparison of Impeller Wall Temperatures (AD / CHT)
In addition to quantitative temperatures, the qualitative distribution also differs. In the adiabatic model, hub temperatures are too low, shroud temperatures are too high and there is a non-existent maximum at the shroud cavity outlet (at impeller inlet). There, the heat flux through the shroud disk is impeded by the hot leakage streaming along the shroud disk (Fig. 6, 5) in the main channel. Therefore, after the shroud's labyrinth seal, heat from the shroud cavity is almost exclusively transported to the IGV by the casing.

The temperature differences between the adiabatic- and the CHT-model result in different temperature gradients and therefore in (possibly) false thermal strain and stress predictions. Since fluid properties are temperature dependant, the differences between the models reveal secondary effects.

\section{Secondary effects}

The kinematic viscosity and thermal conductivity of air rise with fluid temperature, while density decreases. Therefore, frictional losses should increase with higher viscosity at higher temperatures. On the other hand, leakage flow through labyrinths decreases with lower fluid density and higher viscosity. As a result, the amount of leakage that flows through the shroud cavities is slightly higher in the CHT case.

The different leakage flows through the cavities affect axial thrust and disk torque (Hu et al., 2017 and Sun et al., 2009). Due to the differences in temperature and leakage, the combined frictional losses of the cavities in the CHT model are $2-5 \%$ higher than in the adiabatic model. This effect is more evident at higher circumferential Mach numbers. Figure 15 shows the direct comparison of combined frictional power of all impeller cavities as a percentage of total mechanical input power at the shaft.

Differences in axial thrust can also be observed for higher circumferential Mach numbers, but are negligible in this case and will not be presented.

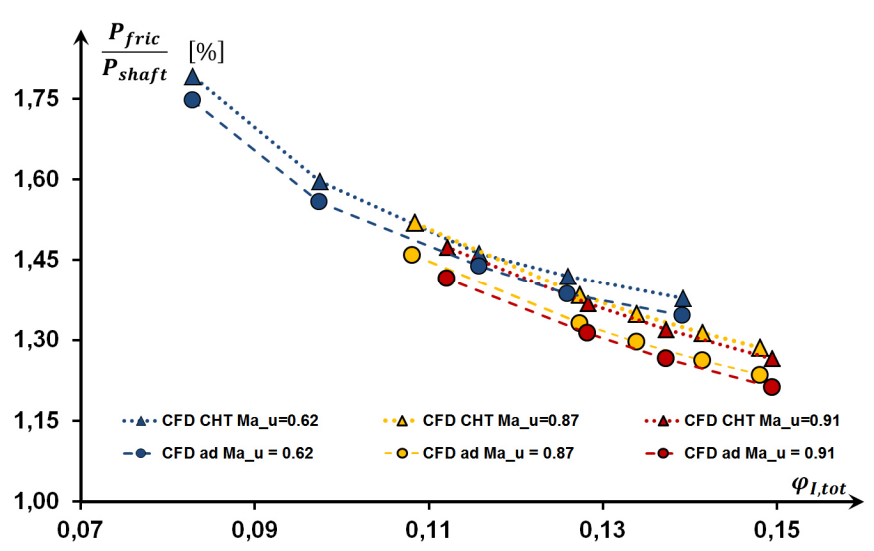

Figure 15 Comparison of Combined Frictional Losses of the impeller cavities (CHT/adiabatic) as Percentage of Shaft Work Input 


\section{CONCLUSIONS}

A CHT calculation of an industrial single stage centrifugal compressor with rotor stator cavities was performed. Additionally, the same fluid mesh was used for a calculation with adiabatic walls. Both models were compared and validated by experimental data.

The adiabatic model proves to be sufficient for reproducing the general characteristics of the centrifugal compressor stage. Main flow bulk temperatures, as well as velocity- and pressure profiles do not differ significantly. But especially in rotor-stator cavities, the adiabatic model yields unacceptable differences, compared to experimental data.

External heat flux at the casing surfaces only amounts to a small percentage of mechanical work input and as a result, the CHT mainly results in a redistribution of thermal energy inside the system. The CHT model shows the most impact on wall temperatures and fluid temperatures in rotor stator cavities. It produces significantly reduced cavity temperatures and agrees well with the measurements.

Secondary effects, resulting from temperature dependant fluid properties, influence disk friction and, to a smaller extent, axial thrust. Especially for low flow number stages this could be more prominent and is worth to be investigated.

Wall temperatures in both models differ significantly regarding temperature levels and distribution. Therefore, the adiabatic model produces completely different temperature gradients, which can be crucial for the structural mechanical design of a compressor.

Regarding the modelling effort, the CHT imposes a significantly higher workload, although computational time was not negatively affected. To the contrary, better convergence of the CHT model could be observed due to the temperature damping effects of the solid bodies - a more physically accurate description of the real compressor.

Concluding, a CHT model should be considered for centrifugal compressor CFD, if fluid temperatures or accurate body temperature predictions are crucial. In those cases, the impeller and the casing bodies close to the cavities should be included in the model. For the sole aerodynamic evaluation of overall efficiency, pressure-, or temperature ratio, the adiabatic approach suffices.

\section{REFERENCES}

Barabas, B., Clauss, S., Schuster, S., Benra, F.-K. and Dohmen, H.J (2015). Experimental and numerical determination of pressure and velocity distribution inside a rotor-stator cavity at very high circumferential Reynolds numbers. Proceedings of the $11^{\text {th }}$ European Conference on Turbomachinery, ETC2015-097.

Berkovsky, B. M. and Polevikov, V. K. (1977). Numerical study of problems on high-intensive free convection. In: Heat transfer and turbulent buoyant convection Vol. 1 and 2., ed. Spalding, D. D., Afghan, H. Washington: Hemisphere. pp. 443-455.
Bohn, D., Heuer, T. and Kusterer, K. (2005). Conjugate Flow and Heat Transfer Investigation of a Turbo Charger. Journal of Engineering for Gas Turbines and Power 127, pp. 663669. doi: 10.1115/1.1839919.

Borm, O., Balassa, B. and Kau, H.-P. (2009). Comparison of Different Numerical Approaches at the Centrifugal Compressor RADIVER. 20th ISABE Conference, International Society for Airbreathing Engines 127. ISABE2011-1242 (2011).

Churchill, S.W. and Chu, H.H.S. (1975). Correlating equations for laminar and turbulent free convection from a horizontal cylinder. Int. J. Heat Mass Transfer 18: pp. 10491053 doi:10.1016/0017-9310(75)90222-7

Daily, J. W. and Nece, R. E. (1960). Chamber Dimension Effects on Induced Flow and Frictional Resistance of Enclosed Rotating Disks. ASME. Journal of Basic Engineering; 82(1), pp. 217-230. doi:10.1115/1.3662532.

Guidotti, E., Naldi, G., Tapinassi, L. and Chockalingam, V. (2012). Cavity Flow Modeling in an Industrial Centrifugal Compressor Stage at Design and Off-Design Conditions. ASME. Turbo Expo: Power for Land, Sea and Air. Turbomachinery, Parts A, B and C Volume 8. GT201268288, pp. 593-603. doi: 10.1115/GT2012-68288.

Hu, B., Brillert, D., Dohmen, H. J. and Benra F.-K. (2017). Investigation on the Flow in a Rotor-Stator Cavity with Centripetal Through-Flow. International Journal of Turbomachinery Propulsion and Power 2017, 2, 18. doi:10.3390/ijtpp2040018

Kluck, N., Geller, M., and Magiera R. (2006). Numerical Computation of Heat Transfer Coefficients for a Radial Compressor Stage using CFD and Comparison with Analytical Model Computations. 24 ${ }^{\text {th }}$ CADFEM User's Meeting 2006 - International Congress on FEM Technology. Web: https://www.fh-dortmund.de/de/fb/5/forschung/ fspcomputersim/medien/pdfs/ThermischeSimulation_S.pdf Access: 03.09.2018.

Lei, V. and Kawakubo, T. (2007). A Fast Method for Conjugate Heat Transfer Analysis of Centrifugal Compressor. ASME International Mechanical Engineering Congress and Exposition. Heat Transfer, Fluid Flows, and Thermal Systems, Parts A and B Volume 8. IMECE200741368, pp. 699-706. doi: 10.1115/IMECE2007-41368.

Lewandowski, W., Klugmann-Radziemska, E. (2001). Heat transfer by free convection from an isothermal vertical round plate in unlimited space. Applied Energy. 68. pp. 187-201. doi:10.1016/S0306-2619(00)00053-2.

Mischo, B., Ribi, B., Seebass-Linggi, C. and Mauri, S. (2009). Influence of Labyrinth Seal Leakage on Centrifugal Compressor Performance. ASME. Turbo Expo: Power for Land, Sea, and Air, Volume 7: Turbomachinery, Parts A and B ():1283-1293. doi:10.1115/GT2009-59524. 
Span, R. (2006). Stoffwerte von Luft. In: VDI Wärmeatlas, ed. Verein Deutscher Ingenieure, VDI-Gesellschaft Verfahrenstechnik und Chemieingenieurwesen (GVC). Berlin, Heidelberg: Springer. Dbb1-Dbb16

Sun Z., Tan C. and Zhang, D. (2009). Flow Field Structures of the Impeller Backside Cavity and its Influences on the Centrifugal Compressor. ASME. Turbo Expo: Power for Land, Sea, and Air, Volume 7: Turbomachinery, Parts A and B ():1349-1360. doi:10.1115/GT2009-59879.

\section{NOMENCLATURE}

\section{Abbreviations}

$\begin{array}{ll}\text { AD } & \text { Adiabatic } \\ \text { CFD } & \text { Computational Fluid Dynamics } \\ \text { CHT } & \text { Conjugate Heat Transfer } \\ \text { Exp. } & \text { Experimental } \\ \text { HTC } & \text { Heat Transfer Coefficient } \\ \text { IGV } & \text { Inlet Guide Vane } \\ \text { LA } & \text { Impeller } \\ \text { RC } & \text { Return Channel }\end{array}$

\section{Dimensionless Quantities}

$\begin{array}{ll}\mathrm{Gr} & \text { Grashof number } \\ \mathrm{Ma} & \text { Circumferential Mach number } \\ \mathrm{Pr} & \text { Prandtl number } \\ \mathrm{TU} & \text { Turbulence intensity } \\ \varphi_{I, t o t} & \text { Flow number }\end{array}$

\section{Non-Dimensionless Quantities}

$\begin{array}{lll}a_{s, I, t o t} & \text { Speed of sound at inlet (total) } & {[\mathrm{m} / \mathrm{s}]} \\ D_{2} & \text { Impeller outer Diameter } & {[\mathrm{m}]} \\ g & \text { Gravitational constant (=9.81) } & {\left[\mathrm{m} / \mathrm{s}^{2}\right]} \\ l_{c} & \text { Characteristic length } & {[\mathrm{m}]} \\ \dot{m} & \text { Massflow } & {[\mathrm{kg} / \mathrm{s}]} \\ P_{\text {fric }} & \text { Frictional power (at disk) } & {[\mathrm{kW}]} \\ P_{\text {shaft }} & \text { Mechanical work } & {[\mathrm{kW}]} \\ \overrightarrow{\vec{q}} & \text { Heat flux density } & {\left[\mathrm{W} / \mathrm{m}^{2}\right]} \\ \dot{Q}_{\text {Casing }} & \text { Heat flux (cavity to stator walls) } & {[\mathrm{kW}]} \\ \dot{Q}_{\text {Rotor }} & \text { Heat flux (cavity to rotor walls) } & {[\mathrm{kW}]} \\ T_{\text {amb }} & \text { Ambient temperature } & {[\mathrm{K}]} \\ u_{2} & \text { Impeller circumferential speed } & {[\mathrm{m} / \mathrm{s}]} \\ \dot{V}_{I, t o t} & \text { Total volume flow at stage inlet } & {\left[\mathrm{m}^{3} / \mathrm{s}\right]} \\ \beta_{v} & \text { Isobaric expansion coefficient } & {[1 / \mathrm{K}]} \\ \Delta T & \text { Driving temperature difference } & {[\mathrm{K}]} \\ v & \text { Kinematic viscosity (fluid) } & {\left[\mathrm{m}^{2} / \mathrm{s}\right]} \\ \lambda_{S} & \text { Thermal conductivity (solid body) } & {[\mathrm{W} / \mathrm{m} \mathrm{K}]}\end{array}$

\section{Indices}

2

cyl

circ

E

I

stat

tot
Impeller outlet

Cylindrical

Circular

Stage exit

Stage inlet

Static (flow)

Total (flow)

Normalized (by overall maximum value)

\section{ACKNOWLEDGMENTS}

The authors gratefully acknowledge the MAN Energy Solutions SE and the German Federal Ministry of Economics and Technology (BMWi). The work was conducted within the funding programme Cooreflex-turbo 1.1.4 'Experimental and numerical investigation of side cavities in centrifugal compressors' (03ET7020R)

\section{APPENDIX A - COPYRIGHT/OPEN ACCESS}

The GPPS policy is that all articles will be Open Source accessible. All articles will be published using the Creative Commons Attribution CC-BY 4.0 or, if commercial rights

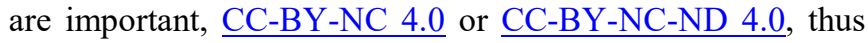
allowing the author(s) to retain their copyright.

For answers to frequently asked questions about Creative Commons Licences, please see https://creativecommons.org/faq/.

\section{APPENDIX B - GPPS PRESENTER POLICY AND PAPER ACCEPTANCE}

According to GPPS's presenter attendance policy, a paper cannot be published or be indexed and may not be cited as a published paper until at least one author pays the registration fee and attends the conference. The GPPS reserves the right to withdraw from its publications any paper that is not presented by an author of the paper at the appropriate conference. Any paper that is withdrawn may not be cited as a published paper.

\section{APPENDIX C - MESH DEPENDENCY}

To show mesh independency, several values of interest between mesh I (16 million elements) and mesh II (40 million elements) are compared in Fig. C.1. Since the finest mesh II showed slight oscillations of some datapoints (e.g. efficiency) in the stationary solution, the maximum instantaneous differences are shown. The differences of averaged values are therefore lower.

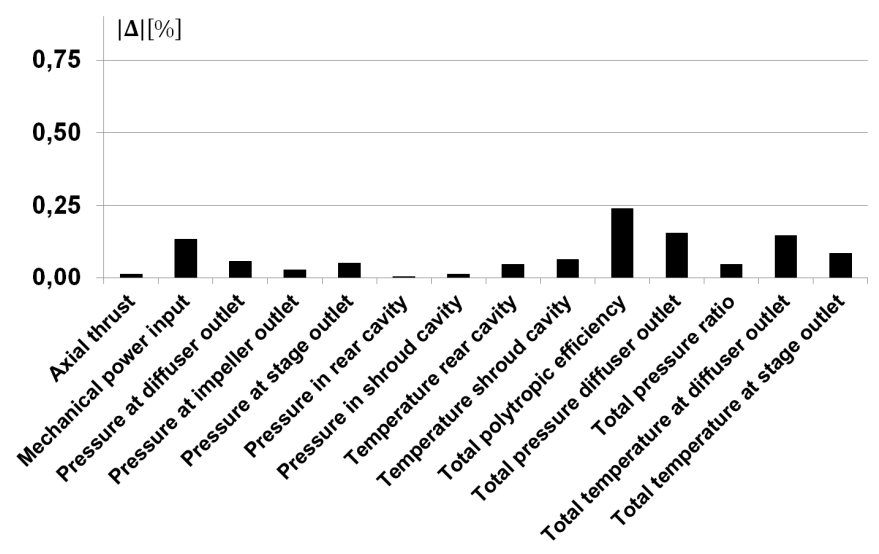

Figure C.1 Comparison of absolute percental differences of several values of interest between mesh I (16 MM. elements) and mesh II (40 MM. elements). 